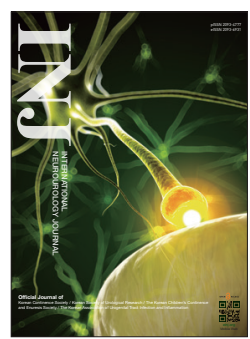

\title{
Interstitial Cystitis/Bladder Pain Syndrome: A Urologic Mystery
}

\author{
Young Sam Cho (iD http://orcid.org/0000-0002-2966-7971 \\ Department of Urology, Kangbuk Samsung Hospital, Sungkyunkwan University School of Medicine, Seoul, Korea \\ E-mail: choys1011@naver.com
}

Interstitial cystitis/bladder pain syndrome (IC/BPS) is a chronic disease characterized by a prolonged duration of symptoms of pelvic or perineal pain, thinning of the bladder epithelium, and various voiding symptoms such as nocturia, increased urinary frequency, and urgency. IC significantly reduces the quality of life and has long been a major burden for patients. The Hunner lesion was originally named "IC" about 100 years ago [1]. It is a discrete area of inflammation that involves the interstitial bladder tissue. Similar to radiation cystitis and bacterial cystitis, the Hunner lesion is also a specific condition that can cause painful bladder symptoms. Most of the issues regarding IC/BPS are controversial, including its definition, prevalence, etiology, pathology, and effective treatment, and many aspects of the diagnosis and definition have been changed since the first report of Hunner.

In 2008, the European Society for the Study of Interstitial Cystitis group published a paper defining BPS as follows:

BPS would be diagnosed on the basis of chronic pelvic pain, pressure, or discomfort perceived to be related to the urinary bladder, accompanied by at least one other urinary symptom like persistent urge to void or urinary frequency. Confusable diseases as the cause of the symptoms must be excluded. Further documentation and classification of BPS might be performed according to findings at cystoscopy with hydrodistention and morphological findings in bladder biopsies. The presence of other organ symptoms as well as cognitive, behavioral, emotional, and sexual symptoms should be addressed [2]. In this definition, the diagnosis of IC/BPS is based on symptoms only, and has become a diagnosis of exclusion. Many diseases might cause bladder pain, and an evaluation was suggested to define such confusable diseases and how they are excluded. Thus, the path to a final diagnosis of IC/BPS is very complicated and mysterious to physicians.

Pathologic changes in IC/BPS are as an important question to physicians as etiology, definition, and treatment. Experimental evidence indicates that a component in the cell coat of the vesical epithelium, a polysaccharide named glycosaminoglycan (GAG), regulates urothelial permeability and prevents noxious compounds in the urine from infiltrating into the bladder wall. Because GAG in the urothelial coating may be defective or even lost in IC/BPS patients, as recently shown [3], urinary compounds could infiltrate into the vesical wall and trigger inflammatory reactions and/or nerve stimulation that would ultimately account for most of the clinical manifestations of IC/ BPS [3,4].

You et al. [5] reviewed recent efforts in the generation of several types of "omics" data sets that have provided an unprecedentedly detailed view of certain diseases, and described available data sets relevant to IC/BPS, such as transcriptome profiles and urinary metabolites as disease biomarkers.

Most of us rely heavily on synoptic manuscripts, such as review articles, to find answers to important questions, with the hope of saving search time; this is a very familiar approach to urologists, especially in a controversial disease such as IC/BPS.

The present review [6] addresses history and changes in the definition, terminology, and diagnostic approach of IC/PBS, and summarizes the histologic findings. Some new pathologic suggestions and urinary markers are also briefly mentioned. 


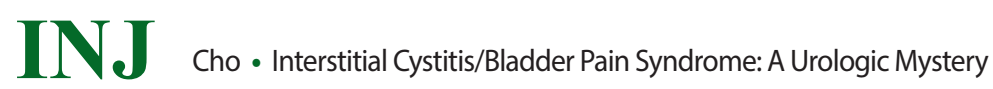

Without understanding of molecular mechanisms and pathology, we cannot resolve the mystery of diagnosis and treatment of IC/BPS. We expect that comprehensive understanding of many aspects of IC/BPS, including the pathology, will be helpful to patients and physicians dealing with IC/BPS.

- Conflict of Interest: No potential conflict of interest relevant to this article was reported.

\section{REFERENCES}

1. Hunner GL. A rare type of bladder ulcer in women; report of cases. Boston Med Surg 1915;172:660-4.

2. van de Merwe JP, Nordling J, Bouchelouche P, Bouchelouche K, Cervigni M, Daha LK, et al. Diagnostic criteria, classification, and nomenclature for painful bladder syndrome/interstitial cystitis: an ESSIC proposal. Eur Urol 2008;53:60-7.

3. Hauser PJ, Dozmorov MG, Bane BL, Slobodov G, Culkin DJ, Hurst RE. Abnormal expression of differentiation related proteins and proteoglycan core proteins in the urothelium of patients with interstitial cystitis. J Urol 2008;179:764-9.

4. Parsons CL. The role of the urinary epithelium in the pathogenesis of interstitial cystitis/prostatitis/urethritis. Urology 2007;69(4 Suppl):9-16.

5. You S, Yang W, Anger JT, Freeman MR, Kim J. 'Omics' approaches to understanding interstitial cystitis/painful bladder syndrome/ bladder pain syndrome. Int Neurourol J 2012;16:159-68.

6. Kim HJ. Update on the pathology and diagnosis of interstitial cystitis/bladder pain syndrome: a review. Int Neurourol J 2016;20:13-17. 